\title{
Autonomic-somatic relationships in the rabbit (Oryctolagus cuniculus): Effects of hippocampal lesions
}

\author{
D. A. POWELL and SHIRLEY BUCHANAN \\ Neuroscience Laboratory, Wm. Jennings Bryan Dorn Veterans' Hospital, Columbia, South Carolina 29201 \\ and University of South Carolina, Columbia, South Carolina 29201
}

\begin{abstract}
Rabbits received sham, cortical control, or dorsal hippocampal lesions and were subjected to simple Pavlovian conditioning. Eyeblink (EB), electromyographic (EMG), and heart rate (HR) CRs were assessed. Shock thresholds, HR URs, and free-field activity were also measured in selected animals. The acquisition of the EB and EMG CRs was not impaired in hippocampal lesioned animals, although hippocampal lesioned animals revealed impaired extinction performance on these measures. The magnitude of the HR CR was enhanced in hippocampectomized animals relative to control animals. Free-field activity was also greater in hippocampal lesioned animals, but shock thresholds and HR URs were unaffected by hippocampectomy. These findings suggest that "orienting" mechanisms may be impaired in hippocampal lesioned animals, resulting in an enhanced visceromotor response to stimulation which, under certain conditions, may affect somatomotor behaviors.
\end{abstract}

When organisms are exposed to a set of learning contingencies, a vast array of physiological and behavioral changes may occur, only one of which is usually of interest in any given learning experiment. However, it is possible that different classes of physiological responses may reflect different stages of the learning process. Thompson, Berger, Cegavske, Patterson, Roemer, Teyler, and Young (1976) recently stressed the importance of separately studying the individual stages of the learning process in an effort to assess the relative neural mechanisms involved. Physiological indices of different stages of learning might be important information in this regard. Although it is obviously prohibitive to measure all physiological changes associated with a particular learning task, a logical analysis might lead to a meaningful small number of response systems which, when taken together, would reflect a fairly complete picture of the learning process.

Experiments in which multiple response systems were assessed have been performed, but primarily to determine the congruence of different response classes in subjects subjected to a variety of experimental manipulations (Black \& deToledo, 1972; Lacey \& Lacey, 1974; Obrist, 1976; Powell \& Kazis, 1976; Schneiderman, 1972). Although the results of such experiments are not completely unequivocal (Elliot, 1974), many of these data suggest that vis-

This research was supported by VA Institutional Research Funds awarded to the Wm. Jennings Bryan Dorn Veterans' Hospital. The authors thank Jack News and Angela LaCombe for their assistance in data collection. Request reprints from D. A. Powell, Neuroscience Laboratory, Wm. Jennings Bryan Dorn Veterans' Hospital, Columbia, South Carolina 29201. ceroautonomic events may reflect an early stage in the learning process (i.e., that stage during which a stimulus assumes its signaling properties) (Graham \& Clifton, 1966; Lacey \& Lacey, 1974; Powell, Mankowski, \& Buchanan, 1978; Pribram \& McGuinness, 1975; Sokolov, 1963), while the somatomotor events, measured in most learning experiments, obviously reflect the end stages of learning during which behavior effects changes in environmental contingencies (Powell \& Joseph, 1974).

We have previously suggested that a minimum of at least three separable stages or processes can be distinguished when an organism adapts to a new set of environmental contingencies. These include (1) attention to relevant stimulation (viz, signals), (2) formation of an association between these signals and a reinforcer (engram formation), and (3) the elaboration of a somatomotor response which deals effectively with the reinforcer (Powell et al., 1978). Presumably different CNS mechanisms mediate these different processes. Data from a previous experiment in our laboratory have suggested that the septum might be involved in the initial, or "attention," stage of learning, since septal lesions enhanced the magnitude of Pavlovian-conditioned heart rate (HR) changes in rabbits and simultaneously interfered with differential eyeblink (EB) conditioning by increasing response frequency to the unreinforced CS- (Powell, Milligan, \& Buchanan, 1976). It was suggested that septal lesions might prevent the normal habituation of the orienting reflex (OR) (Graham \& Clifton, 1966; Sokolov, 1963), resulting in an increase in responding to irrelevant or nonreinforced stimulation. Similar models of the function of the closely related dorsal 
hippocampus have been postulated (Douglas, 1972; Pribram \& McGuinness, 1975; Vinogradova, 1975). The present investigation sought to further assess this "attention model" of the septal-hippocampal system by studying the effects of lesions of the dorsal hippocampus on Pavlovian heart rate conditioning.

Several studies have shown that the OR is affected by hippocampal lesions (Crowne \& Riddell, 1969; Sanwald, Porzio, Deane, \& Donovick, 1970); Pavlovian somatomotor conditioning has also been studied in animals with hippocampal damage (Schmaltz \& Theios, 1972; Solomon, 1977; Solomon \& Moore, 1975). However, the cardiac CRs which accompany such conditioning have not been studied. Thus, the major purpose of the present experiments was to examine the effects of hippocampal lesions on Pavlovian HR conditioning. Specifically, it was hypothesized that (1) if the Pavlovian bradycardiac HR CR reflects an attention process, and (2) if hippocampal lesions affect behavior by disruption of an attention mechanism, then hippocampal lesions should affect HR conditioning. Based on the results of lesions of the septal region (Powell et al., 1976), it was further believed that hippocampal lesions would increase the magnitude of the HR CR. Although the major interest in the present experiments was on the HR CR, eyeblink (EB) and electromyographic (EMG) CRs were also recorded in order to relate any cardiovascular changes produced by hippocampal lesions to possible changes in somatomotor response systems. In addition, two control experiments were performed to assess whether sensory or motor impairments might be produced by hippocampal damage and thus mediate the changes in conditioning produced by the lesions.

\section{EXPERIMENT 1}

\section{Method}

Animals. The animals used were New Zealand albino rabbits purchased locally. The 24 rabbits were divided into three groups of 8 subjects each. One group received hippocampal lesions, the second, cortical control lesions, and the third, sham lesions. The subjects weighed approximately $2.5 \mathrm{~kg}$; half were males and half were females. All animals were maintained on ad-lib food and water in a holding room with a 7 a.m. on - 7 p.m. off light/dark cycle. All experiments were conducted during the daylight portion of the $\mathrm{L} / \mathrm{D}$ cycle.

Apparatus. The rabbits were restrained in standard Plexiglas rabbit restrainers within sound-attenuated experimental chambers that consisted of ventilated refrigeration shells. The ECG electrodes were stainless steel safety pins that were inserted beneath the skin on the right front leg and left haunch. Insertion of these pins appeared to be harmless; no infection or evidence of discomfort was observed. Stainless steel eye clips inserted beneath the upper and lower eyelids served as electrodes for recording the corneoretinal potential (CRP) or EB response. This reaction, described in more detail in VanDercar, Swadlow, Elster, and Schneiderman (1969) consists of a response constellation that includes eyelid closure, extension of the nictitating membrane, and retraction of the eyeball. EMG activity was measured by inserting previously prepared insect pins into the neck muscles.
All EB, HR, and EMG responses were measured on a Grass Model 5 polygraph. The shock US was administered through stainless steel Michel wound clips chronically inserted approximately $1 \mathrm{~cm}$ above and below the right eye. The tone CS was produced by a Bud Code Practice oscillator and was presented to the subject through $15 \times 23 \mathrm{~cm}$ speakers situated $30 \mathrm{~cm}$ above the animal's head. Electronic timers in conjunction with BRS solid state programming equipment automatically programmed the events of the experiments. The recording and control equipment was located in a room adjacent to the experimental chambers.

Surgery and Histology. All subjects were anesthetized with ketamine hydrochloride $(55 \mathrm{mg} / \mathrm{kg})$ and chlorpromazine hydrochloride $(10 \mathrm{mg} / \mathrm{kg})$ administered $\mathrm{im}$. The animal was placed in a Kopf stereotaxic instrument, and the skull was exposed by a midline incision. Using a dental drill, the bone was removed from $1 \mathrm{~mm}$ posterior to bregma to 3 to $4 \mathrm{~mm}$ anterior to the lambdoid suture. A thin 1-2-mm strip of bone was left intact overlying the superior sagittal sinus. For the sham control animals, the skin overlying the scalp was closed with wound clips and the animal was returned to its home cage. For the remaining subjects, the dura was cut with an iris scissor and the surface of the cortex overlying the hippocampus was exposed. The cortex and white matter overlying the dorsal hippocampus was removed by subpial aspiration, taking care not to damage the dorsal hippocampus in cortical control subjects. Subjects in the hippocampal groups were treated similarly, but, in addition, the dorsal hippocampus and, to varying degrees, the posteroventral hippocampus were removed. In both cortical control and hippocampal animals, a small amount of Gelfoam was placed in the incision to hasten clotting. After all bleeding had ceased, the incisions were closed with wound clips. All animals were administered a prophylactic injection of 200,000 units of Bicillin. In addition, all subjects were maintained on tetracycline antibiotic for a period of 2 weeks postsurgery. At the conclusion of the study, all subjects were injected with a lethal dose of sodium pentobarbital; the heart was rapidly exposed and perfused with a $.9 \%$ saline solution followed by a $10 \%$ Formalin solution. Approximately 1 week later, frozen sections were taken through the lesion at $\mathbf{4 0}$ microns. Every fifth section was stained with hematoxylin and counterstained with Oil Red-O for histological verification of the extent of hippocampal and cortical damage.

Procedure. Approximately 3 weeks following surgery, behavioral testing began. During the first experimental session (adaptation), the animals were exposed to 64 presentations of the $\mathrm{CS}$ alone. Consecutive acquisition sessions separated by $24 \mathrm{~h}$ followed until the subject had reached a criterion of $80 \%$ responding during 3 consecutive sessions, at which time extinction training began. During extinction sessions, the CS was again presented alone for two consecutive daily sessions. A constant 90 -sec intertrial interval was employed. The CS was a 75-dB (SPL), 1-secduration tone with a frequency of $1,216 \mathrm{~Hz}$. A 5-mA paraorbital ac electric shock was the US. US duration was $.5 \mathrm{sec}$ with no delay between CS offset and the US onset. Several interspersed presentations of each tone per session served as test trials, during which HR was recorded to the tone alone since the CS was not followed by shock on these trials. On each test trial, a baseline measurement consisted of the mean duration of 10 heartbeats immediately preceding CS onset. The duration of 30 beats following CS onset was then compared with this baseline in blocks of 5-beat intervals. EB and EMG responses were recorded during each CS presentation. These responses were defined as a $200-\mu \mathrm{V}$ deflection of the appropriate recording pen during the CS period. At the gain setting used on the preamplifier of the polygraph, this deflection was equivalent to an EB response of $2 \mathrm{~mm}$ or greater.

\section{Results}

Histology. Histological analysis of the brain sections revealed that the dorsal hippocampus was removed bilaterally in all animals in the hippocampal lesioned 


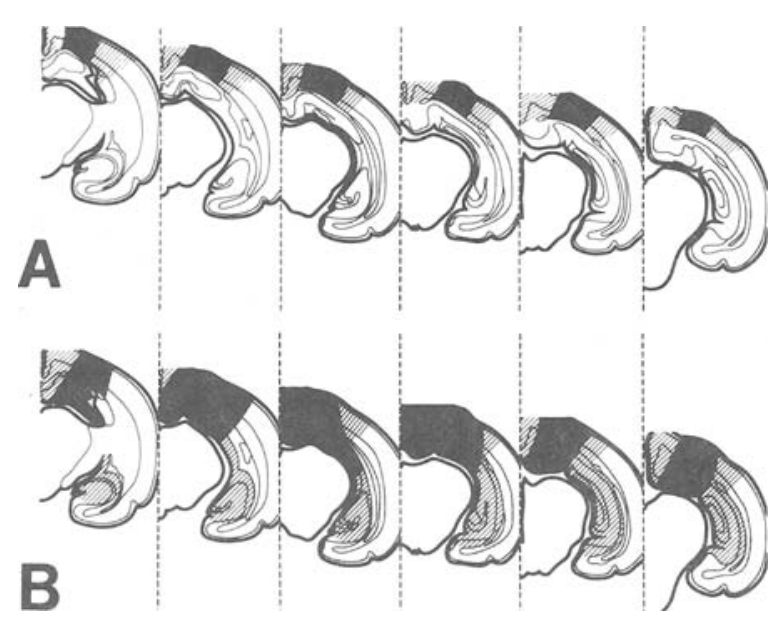

Figure 1. Maximal (striped) and minimal (crosshatched) damage to (A) cortical lesioned and (B) hippocampal lesioned rabbits which received simple Pavlovian conditioning training.

group. The posteroventral portion of the hippocampus was intact in all but two animals, although the fimbria was bilaterally sectioned in all hippocampal lesioned animals. Minimal and maximal damage is shown in Figure 1 for (A) cortical control and (B) hippocampal subjects. In no animals of the former group was the dorsal hippocampus damaged. Damage to other structures was minimal. In two hippocampal lesioned subjects, there was slight unilateral damage to the body of the caudate nucleus, but these subjects did not appear to be atypical behaviorally, compared with the other animals in the hippocampal lesioned group. No animals revealed dorsal thalamic damage.

Heart rate. The HR data are shown in Figure 2. Pretone baseline data as well as the mean change from pretone baseline during the first five blocks of beats are shown separately for adaptation, acquisition, and extinction training, respectively. Since the animals were trained to an EB criterion, different subjects were exposed to varying numbers of acquisition sessions. Thus, the acquisition data are shown only for the first, middle, and last sessions for each group. Mean tonic heart rate (as evaluated during the pretone period) revealed a U-shaped function for all groups of animals; viz, baseline heart rate was faster during the first and last acquisition sessions than during the middle sessions of the experiment. These changes in baseline HR support previous findings (Powell \& Milligan, 1975). Change from pre-CS baseline was an HR decrease in all animals. The most significant aspect of these data was the fact that the increase in magnitude of HR deceleration in the hippocampectomized animals was more than double that in the control animals. During adaptation and extinction, the hippocampal lesioned animals also revealed faster baseline $\mathrm{HR}$ and greater change from HR baseline than did the cortical control or sham operated animals.
The acquisition HR data were subjected to a mixeddesign analysis of variance (ANOVA) utilizing as variables (1) the surgical conditions (viz, groups, 3 levels), (2) sessions (3 levels), and (3) pretone vs. posttone ( 2 levels). This analysis revealed that the effects of training sessions and pre- vs. posttone were significant $[\mathrm{F}(2,42)=10.9, \mathrm{p}<.01 ; \mathrm{F}(1,21)=14.2$, $\mathrm{p}<.01]$. The group variable was also significant $[F(2,21)=4.8, p<.025]$, and groups interacted with sessions $[\mathrm{F}(4,42)=5.2, \mathrm{p}<.01]$. A post hoc analysis of HR difference scores (viz, HR pre minus HR post), using the Duncan multiple range test, revealed that hippocampal lesioned animals showed greater HR changes than either the sham operated or control animals during all three sessions $(\mathrm{p}<.01)$. However, the latter groups did not differ from each other. The second block of five post-CS heartbeats (not shown in Figure 2) was also significantly larger in the hippocampal lesioned animals, although the remaining blocks of beats (viz, 3-6) were not. A separate analysis of baseline heart rate (pretone) revealed no significant effects among animals with different lesions; however, the sessions effect was significant $[F(2,42)=8.45$, $\mathrm{p}<.01]$. ANOVAs conducted on the adaptation and extinction data revealed that the groups also differed during extinction, with the hippocampal animals showing a slower HR baseline $[\mathrm{F}(2,21)=3.5, \mathrm{p}<.05]$ as well as greater $H R$ changes from baseline $[F(1,21)=$ $4.62, \mathrm{p}<.01]$ than the cortical control or sham operated animals. Only the sessions effect was significant during adaptation $[F(1,21)=3.81, p<.05]$.

Eyeblink and EMG. Mean EB and EMG percent responses are shown in Figure 3 for the first, the middle, and last acquisition sessions for each group of animals. Data are also shown for adaptation (ADP) and extinction (EXT). These data represent the percent of total trials during each session on which an EB or EMG CR, as previously defined, occurred. No group differences occurred during adaptation. Similarly, there were few differences between the

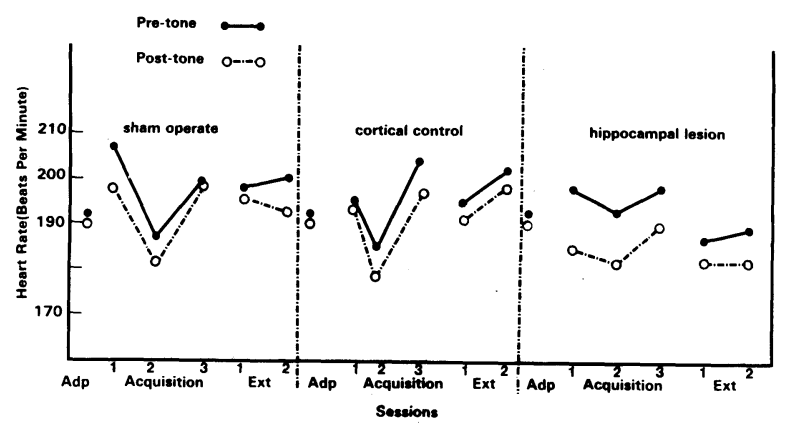

Figure 2. Heart rate CRs (open circles) and pre-CS heart rate baseline (closed circles) of three groups of rabbits which were exposed to simple Pavlovian conditioning training as shown. Data are shown for (a) one adaptation (ADP) session, (b) the first, middle, and last acquisition sessions, and (c) two extinction (EXT) sessions. 


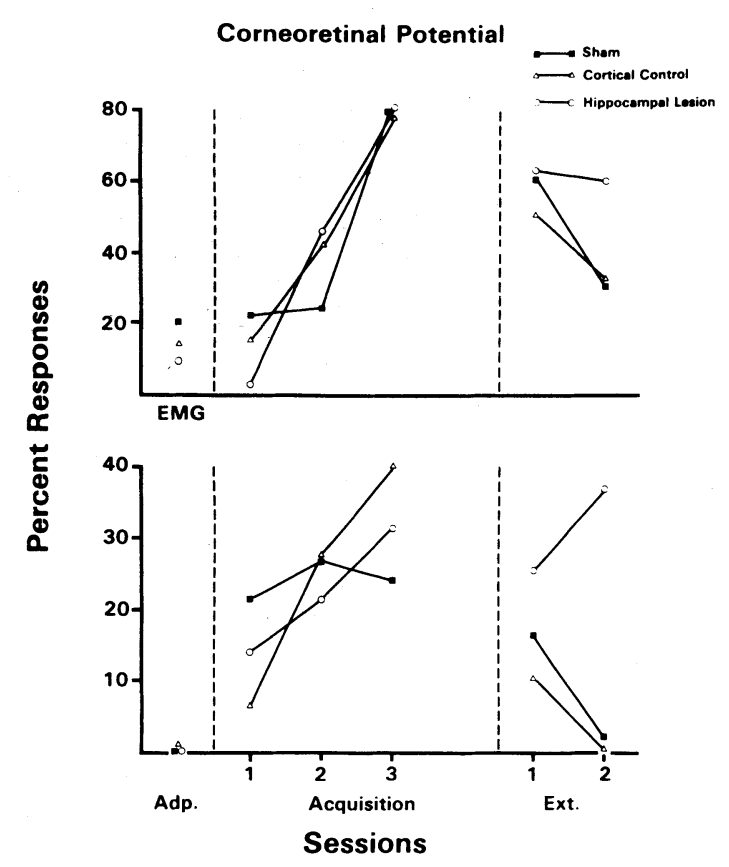

Figure 3. Percent corneoretinal potential (viz, eyeblink) and EMG CRs during adaptation (ADP), acquisition, and extinction (EXT) of three groups of rabbits exposed to simple Pavlovian conditioning training as shown. Since the subjects were trained to an acquisition criterion (80\% CRP responding) and thus received differing numbers of acquisition sessions, the acquisition data represent the first, the middle, and last days of training for each group of subjects.

groups in the rate of acquisition of either the EB or EMG response. Analysis of variance of the number of sessions to reach the $80 \%$ response criterion revealed no differences between the cortical control, sham, and hippocampal lesioned subjects with regard to the EB response. Mean number of sessions $( \pm 1$ SEM) for hippocampal lesioned animals was 6.4 $( \pm 1.2)$; for cortical control subjects, it was $8.2( \pm 2.1)$; and for sham lesioned subjects, it was $7.4( \pm 1.8)$. However, both EB and EMG CRs in the hippocampal lesioned animals were greater during extinction than those obtained for the cortical control or sham groups. An analysis of variance of the extinction data revealed that the hippocampal lesioned animals showed significantly more EB and EMG CRs during the second extinction session $[\mathrm{F}(2,21)=5.76, \mathrm{p}<.01$, and $\mathrm{F}(2,21)=8.42, \mathrm{p}<.01$, for EB and EMG, respectively]. However, these differences were not significant during the first extinction session.

\section{Discussion}

The HR results of the present experiment are compatible with a previous experiment in which it was shown that septal lesions also enhanced the HR CR in rabbits exposed to a Pavlovian conditioning contingency (Powell et al., 1976). In the present experiment, HR CR magnitude was greater in hippocampal lesioned rabbits than in cortical control or sham operated animals. These findings thus implicate both the septum and dorsal hippocampus in the processing of sensory information associated with Pavlovian conditioning. Such stimulus processing may be highly similar to that associated with the orienting reflex (OR), which also consists of cardiac inhibition (Graham \& Clifton, 1966), and is affected by both septal and hippocampal manipulations (Crowne \& Riddell, 1969; Sanwald et al., 1970). Septal lesions have also been found to attenuate tachycardiac CRs in unrestrained rats (Duncan, 1972; Holdstock, 1970). Although the latter findings may at first glance appear to contradict the present data, the net effect in the latter experiments was also a lesion-induced HR change in a parasympathetic direction. Thus, if one assumes that inhibition in the cardiovascular system is associated with the occurrence of the OR (Graham \& Clifton, 1966; Lacey \& Lacey, 1974; Powell \& Kazis, 1976), these data also suggest a possible interference with orienting mechanisms by septal and/or hippocampal lesions.

The present data do not, however, entirely support previous investigations of Pavlovian somatomotor conditioning. Schmaltz and Theios (1972), who also studied the effects of hippocampectomy on Pavlovian conditioning in rabbits, found that hippocampal lesions produced faster nictitating membrane conditioning than did cortical control or sham lesions. However, the latter investigators used a 250 -msec, ISI, whereas the present study employed a 1-sec ISI. A longer ISI was used in the present experiment because conditioned HR changes require longer ISIs for their optimum development (Powell, Lipkin, \& Milligan, 1974). In addition, the latter investigators used an acquisition criterion of 10 consecutive NMR CRs and employed a larger number of conditioning trials per session. Thus, the lack of support for the findings of Schmaltz and Theios (1972) in the present experiment may have been due to the use of a different set of conditioning parameters. In order to evaluate the present findings within the context of the original experiments of Schmaltz and Theios, the present data were reanalyzed in terms of the number of trials required to reach a criterion of 10 consecutive EB CRs. Although the animals with hippocampal lesions required fewer trials to reach this criterion (95.4 \pm 15.6) than did the cortical control or sham operated animals (164.4 \pm 29.6 and $136.8 \pm 26.8$, respectively), an analysis of variance of these data revealed that they were not statistically significant $[\mathrm{F}(2,21)=$ $3.04, .05<\mathrm{p}<.10$ ]. However, these differences are obviously in the direction predicted by the data of Schmaltz and Theios (1972).

The greater EB and EMG responding during extinction also supports the findings of Schmaltz and Theios (1972). In the latter study, animals with hippo- 
campal lesions did not show an extinction deficit during initial exposure to extinction conditions but, after multiple exposures, rabbits with hippocampal lesions did show increased NMR CRs during extinction compared with either sham or cortical control subjects. Likewise, in the present study, differences in EB CRs were not statistically significant during the first session of extinction, but both EB and EMG CRs were significantly greater in the subjects with hippocampal lesions during the second session. The latter findings are compatible with the contention of Solomon (1977) and Solomon and Moore (1975) that animals with hippocampal lesions show a deficit in latent inhibition. Latent inhibition refers to a decrement in conditioning produced by exposure to the CS unpaired with the US prior to conditioning training. Rabbits with hippocampal lesions did not reveal such decrements (i.e., latent inhibition) in an NMR conditioning task (Solomon \& Moore, 1975), but did show evidence of conditioned inhibition. The latter is analogous to Pavlovian internal inhibition, in which response inhibition is actively conditioned to specific stimuli (Solomon, 1977). Solomon and Moore (1975) suggest that response decrements associated with latent inhibition are due to a "tuning out" process associated with irrelevant stimulation and that hippocampectomized animals are unable to "tune out" such irrelevant stimuli, resulting in an increase in responding compared with control subjects. Intuitively, extinction would appear to involve latent, rather than conditioned, inhibition, since all stimuli during extinction are irrelevant and can safely be tuned out; that is to say, it would be inefficient to actively attend to stimuli signaling nonreinforcement when there are no stimuli present to which it was necessary to direct attention.

Indeed, it is possible that the faster NMR acquisition in hippocampal lesioned subjects reported by Schmaltz and Theios (1972) was related to latent inhibition deficits in these subjects. The subjects in that study, as well as those in the present investigation, received prior CS exposure, which would have delayed acquisition in the normal rabbits, but not in rabbits with hippocampal damage. Such preexposure, usually employed as a control for sensitization and pseudoconditioning, thus may influence the performance of brain-damaged animals when inhibitory processes are mediated by the damaged structures. Although differences in acquisition between hippocampal and control subjects in the present study were not statistically significant, they were in the same direction as those reported by Schmaltz and Theios (1972), that is, faster acquisition in hippocampal lesioned subjects. A major difference between the present experiment and that of Schmaltz and Theios, however, is that $24 \mathrm{~h}$ intervened between CS preexposure (viz, adaptation) and initiation of acquisition training in the present study, whereas prior CS exposure occurred just prior to conditioning in the Schmaltz and Theios study. Thus, the effects of prior CS exposure may have dissipated during the intervening $24 \mathrm{~h}$ in the present study.

Thus, in general, the present findings are in agreement with those of previous investigators. Taken together, these data and those of the previous studies discussed above suggest a possible disruption of an "attention" process in hippocampectomized subjects. Moreover, Solomon's (1977) data suggest that these attentional deficits are represented behaviorally by a deficit in latent inhibition.

\section{EXPERIMENT 2}

The larger HR CRs observed in the lesioned subjects of Experiment 1 suggest that hippocampal lesions may interfere with an attention mechanism related to habituation of the orienting reflex. However, a simpler explanation is that hippocampectomized subjects may simply show enhanced responding to all kinds of sensory stimulation. Other studies, for example, have shown increased locomotor activity in hippocampectomized animals compatible with this interpretation (e.g., Lanier \& Isaacson, 1975). Two control experiments thus sought to further test this hypothesis (1) by measuring the somatomotor and HR thresholds to the paraorbital ac shock used as the US in the first experiment, and then (2) by measuring the locomotor activity of the subjects studied in Experiment 1. The present experiment measured $H R$ and EB thresholds in these animals.

\section{Method}

Animals, Surgery, and Histology. The present experiment was performed after Experiment 1 was completed, using the animals previously used in that experiment.

Apparatus and Procedure. The apparatus was identical to that used in Experiment 1. However, the procedure was a shock threshold procedure in which the method of constant stimuli was used to present six different shock intensities in a random order for 10 presentations each. The 500 -msec shock was presented to electrodes attached above and below the eyelids, as in the previous experiment. However, in the present experiment, the shock was unsignaled. A constant 15-sec intertrial interval was employed. The following measurements were obtained: (1) the magnitude of the unconditioned heart rate change in response to each shock presentation; (2) the frequency of "jumping" (viz, a movement of the entire body); (3) the frequency of the eyeblink response (viz, eyelid closure and nictitating membrane extension without an accompanying jump); and (4) the frequency of a "twitch" of the eye muscles (viz, eyelid or nictitating membrane movement without eyelid closure). The shock thresholds for the latter three responses were defined as the shock intensity that elicited a response $50 \%$ of the time. The shock intensities employed were $0, .1, .2, .5,1.0$, and 2.0 mA. See Powell et al. (1978) for more details regarding this procedure.

\section{Results}

The mean shock intensities (viz, threshold \pm 1 SEM) required to elicit the (1) eyelid twitch, (2) eyeblink reflex, and (3) jumping response are shown in Table 1 
Table 1

Mean Paraorbital Shock Thresholds (in Milliamperes) and Standard Errors for Rabbits with Hippocampal, Cortical, and Sham Lesions

\begin{tabular}{|c|c|c|c|c|c|c|c|}
\hline \multirow[b]{2}{*}{ Lesion } & \multirow[b]{2}{*}{$\mathrm{N}$} & \multicolumn{2}{|c|}{ Twitch } & \multicolumn{2}{|c|}{ Eye Blink } & \multicolumn{2}{|c|}{ Jump } \\
\hline & & Mean & SE & Mean & SE & Mean & SE \\
\hline Hippocampal & 8 & .198 & .11 & .990 & .42 & 2.08 & .47 \\
\hline Cortical & 8 & .171 & .07 & .968 & .70 & 2.02 & .22 \\
\hline Sham & 8 & .226 & .17 & .970 & .42 & 2.38 & .44 \\
\hline
\end{tabular}

Note-See text for definition of responses.

for each of the three groups of subjects as defined in Experiment 1. An analysis of variance of these data revealed no significant differences between the three groups on any response measure. The unconditioned heart rate change was an acceleration in rate that was directly related to shock intensity, as has been reported in previous experiments (Kazis, Milligan, \& Powell, 1973). However, again there were no significant differences between the hippocampal, cortical control, and sham groups of subjects on either baseline HR or change in HR in response to the shock, as determined by a repeated measures analysis of variance. Mean baseline HR was 215.7, 195.8, and 205.7 for the hippocampal, cortical, and sham-lesioned groups, respectively. Mean change from baseline for these groups during the first block of 5 postshock beats in response to the highest intensity employed ( $2 \mathrm{~mA}$ ) was $38.4,43.4$, and $34.8 \mathrm{bpm}$, respectively. Thus, neither somatomotor thresholds nor HR URs in response to paraorbital shock were affected by hippocampal lesions; it is conceivable, however, as noted by Jarrard (1973), that hippocampectomized subjects are more sensitive to relatively higher levels of suprathreshold shock.

\section{EXPERIMENT 3}

\section{Method}

Animals. The rabbits in this experiment also had served as subjects in Experiment 1. Two additional subjects, which had similar lesions and which had previously served in an unpublished classical conditioning experiment very similar to Experiment 1, were also tested in each group; thus, the present experiment used 10 hippocampal lesioned, 10 cortical lesioned, and 10 sham operated animals.

Apparatus and Procedure. Free-field activity was measured in a 91.4-cm-square plywood box that was painted flat black. Photocells mounted in each side of this box divided it into four quadrants. A photocell was activated when the rabbit crossed from one quadrant to an adjacent quadrant. The photocells, in turn, activated a counter which counted the number of quadrants crossed during each 15-minute session. Ten consecutive daily sessions were given, beginning approximately 5 days after surgery and continuing up to the beginning of classical conditioning training.

\section{Results and Discussion}

The mean activity scores of each of the three groups of animals are shown in Figure 4 as a function of experimental sessions. These activity scores represent the mean number of quadrants crossed during each 15-min session for each group. The activity of the hippocampal lesioned subjects gradually increased over the first half of the experiment, attaining values of 143 and 149 in Sessions 5 and 9, respectively. The cortical control group also showed a gradual increase over sessions, but stabilized at a lower level. The sham group's scores, on the other hand, declined over sessions, reaching mean values of from 30 to 40. A repeated measures analysis of variance was computed on these data utilizing as dimensions groups (3 levels) and sessions (10 levels). The groups variable was significant $[F(9,243)=24.6, p<.01]$; the interaction of Groups by Sessions was also significant $[F(18,243)=5.49, \mathrm{p}<.01]$. Thus, as has been found in previous experiments on rats (e.g., Lanier \& Isaacson, 1975), hippocampal lesions produced dramatic increases in general locomotor activity. These results contrast with those obtained in septal lesioned rabbits, which did not show enhanced general activity unless stimulated by intense photic stimulation (Powell et al., 1976). Lanier and Isaacson (1975) also found that there was an increase in activity over the first 10 days in hippocampal lesioned rats, but that activity declined thereafter. The lesioned subjects in the present study showed no evidence of decreasing activity, but they were, of course, observed for only 10 days.

\section{GENERAL DISCUSSION}

Few experiments have investigated Pavlovian conditioning in animals with hippocampal damage. As noted above, Schmaltz and Theios (1972) found that the nictitating membrane $\mathrm{CR}$ was more rapidly acquired by hippocampal lesioned rabbits, while Solomon and Moore (1975) found that hippocampal lesioned

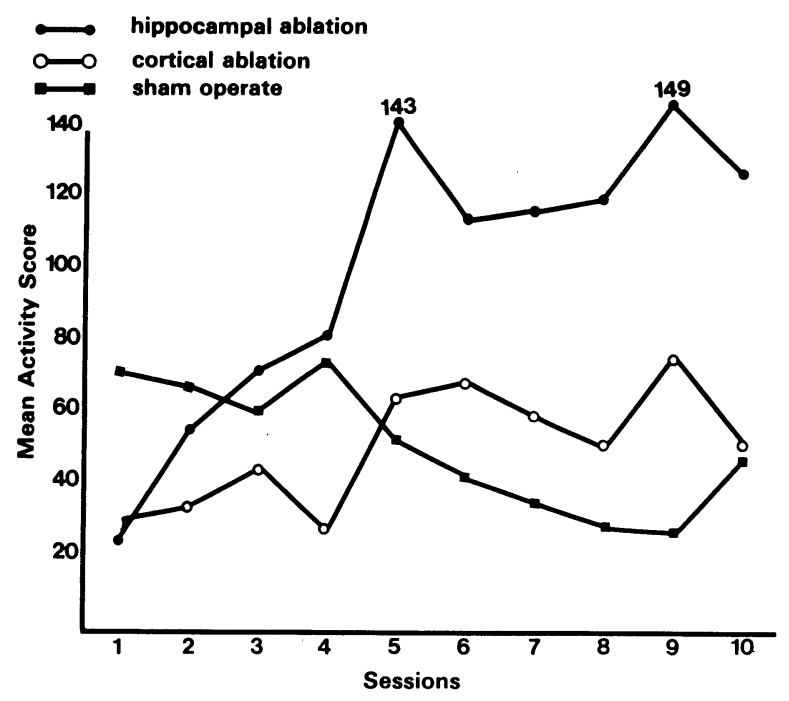

Figure 4. Mean free-field activity scores of rabbits with (a) hippocampal lesions, (b) cortical lesions, and (c) sham lesions as a function of $\mathbf{1 0}$ consecutive daily sessions. 
rabbits did not reveal the acquisition deficits which intact rabbits exhibit when previously exposed to CS-alone presentations. The purpose of the present experiments was to extend the findings of these investigators by testing the hypothesis that Pavlovian conditioned HR CRs would also be affected by hippocampal lesions. This hypothesis was based on the prior observation that septal lesions enhanced the HR CR that accompanies EB conditioning in the rabbit. Since both input and output pathways exist between the septum and hippocampus (Powell \& Hines, 1975), it is reasonable to assume that the larger magnitude HR CRs exhibited by septal lesioned subjects are related to interruption of septal-hippocampal circuits. Moreover, one of the major models of hippocampal function involves its possible participation in the stimulus processing associated with attentional phenomena (Douglas, 1967; Pribram \& McGuinness, 1975). Since cardiac inhibition represents the cardiac component of the "orienting reflex" (Graham \& Clifton, 1966; Powell \& Kazis, 1976), larger magnitude bradycardiac CRs in lesioned animals would suggest that "orienting" or "attentional" dysfunctions might exist in these animals. The results of the present experiments support such an interpretation. The larger magnitude HR CRs obtained in the hippocampal lesioned subjects in Experiment 1 suggest that an orienting or attentional dysfunction may have been produced by the lesion, assuming that decelerative HR changes are indicative of these processes. The failure of locomotor activity to decline over time in the hippocampal lesioned subjects, as it did in the control subjects of Experiment 3, could also be related to a deficit in habituation of the OR to nonsignificant (viz, nonreinforced) stimulation.

A more detailed explanation of these findings is suggested by recent electrophysiological studies of hippocampal function. Vinogradova (1975) reported that hippocampal pyramidal cells show a characteristic firing pattern to all modalities of sensory stimulation. Moreover, the response of these cells bears a systematic relationship to the behavioral changes that occur during orienting and habituation (Sokolov, 1963). Vinogradova (1975) specifically suggests that the hippocampus is involved in the visceroautonomic response to novel stimulation as the "comparator" in a system that is sensitive to novel stimulation (viz, stimuli that stand out from ongoing background stimulation). According to this model of hippocampal function, the hippocampus "compares" all currently incorning stimulation with a "cortical model" of previously ongoing background stimulation. When a mismatch occurs, the output of the hippocampal cells, which were previously inhibiting lower brain-stem centers, is decreased, thus releasing these centers so that the OR occurs (see Pribram \& McGuinness, 1975). Thompson and his colleagues
(Thompson et al., 1976) have recently shown that hippocampal cells also show a pattern of firing that closely follows the elaboration of the classically conditioned NMR in the rabbit to a tone-airpuff contingency. Thompson et al. (1976) suggest, based on these data, that the hippocampus may mediate early events in the process of engram formation. Data from Olds and his colleagues (e.g., Segal \& Olds, 1973) support this suggestion. Hippocampal unit firing has also been related to spatial stimuli associated with novelty and reinforcement in instrumental learning tasks (see O'Keefe and Nadel, 1978, for a recent review). These electrophysiological data are thus also compatible with the hypothesis that the hippocampus is involved in orienting to novel stimulation (i.e., the OR) and in processing information associated with the CS (i.e., the signal) during classical conditioning. If the bradycardia associated with orienting and conditioning reflects such stimulus processing, then the HR changes that accompany both orienting and Pavlovian conditioning may be under tonic inhibitory hippocampal control. Thus, when such inhibition is removed, either by interruption of the septal-hippocampal input pathways to the system, as in the Powell et al. (1976) septal lesion experiment, or by removal of the hippocampal cells proper, as was done in the present experiment, an exaggerated HR CR may be obtained.

The above analysis rests upon the assumption that the visceromotor component of the $C R$ reflects a different kind of behavioral process than does the somatomotor component. Specifically, the HR CR is assumed to be indicative of "orienting" or "attention," while EB and EMG CRs are assumed to reflect the mobilization of somatomotor behaviors in response to an aversive CS-US contingency (also see Izquierdo \& Elisabetsky, 1979, in this regard). As noted above, there are substantial reasons for believing this to be the case. However, an alternative interpretation is that bradycardiac HR CRs are merely accompaniments of generalized somatomotor inhibition. Obrist (1976), for example, suggests that most, if not all, cardiac changes associated with Pavlovian tasks may be mediated by a central "cardiac-somatic" linkage. Even though cardiac decelerations and somatic inhibition may be closely related temporally, a close relationship between $\mathrm{HR}$ and somatomotor changes does not necessarily preclude a prominent role for the cardiovascular system in "attention phenomena"; that is, the "cardiac-somatic linkage" and "attention" models of cardiac-somatic integration are not logically incompatible (also see Lacey \& Lacey, 1974). Hence, behavioral inhibition might also accompany "attentional states," such as orienting, without severely damaging attention models of septalhippocampal function (Powell \& Joseph, 1974). In fact, somatic inhibition might be predicted by the Pribram- 
McGuinness (1975) model of hippocampal function since, according to this model, hippocampal circuits mediate both stimulus registration and response selection mechanisms. Support for these speculations will only obviously require further research in which more sensitive measurements of task-irrelevant somatic activity are made.

\section{REFERENCES}

Black, A. H., \& DeToledo, L. The relationship among classically conditioned responses: Heart rate and skeletal behavior. In A. H. Black \& W. F. Prokasy (Eds.), Classical conditioning II: Current theory and research. New York: Appleton-CenturyCrofts, 1972.

Crowne, D. P., \& Riddell, W. I. Hippocampal lesions and the cardiac component of the orienting response in the rat. Journal of Comparative and Physiological Psychology, 1969, 69, 748-755.

Douglas, R. J. The hippocampus and behavior. Psychological Bulletin, 1967, 67, 416-422.

Douglas, R. J. Pavlovian conditioned inhibition and the brain. In R. A. Boakes \& M. S. Halliday (Eds.), Inhibition and learning. New York: Academic Press, 1972.

Duncan, P. M. Effect of septal area damage and base-line activity levels on conditioned heart-rate response in rats. Journal of Comparative and Physiological Psychology, 1972, 81, 131-142.

ElliotT, R. The motivational significance of heart rate. In P. A. Obrist, A. H. Black, J. Brener, \& L. V. DiCara (Eds.), Cardiovascular psvchophvsiology: Current issues in response mechanisms, biofeedback and methodology. Chicago: Aldine, 1974.

Graham, F. K., \& Clifton, R. K. Heart rate change as a component of the orienting response. Psychological Bulletin, 1966, $65,305-320$.

HolDstock, T. L. Plasticity of autonomic functions in rats with septal lesions. Neuropsychologia, 1970, 8, 147-160.

Izquierdo, I., \& Elisabetsky, E. Physiological and pharmacological dissection of the main factors in the acquisition and retention of shuttle behavior. In M. A. B. Brazier (Ed.), Brain mechanisms in memory and learning. New York: Raven Press, 1979.

Jarrard, L. E. The hippocampus and motivation. Psychological Bulletin, 1973, 79, 1-12.

Kazis, E., Milligan, W. L., \& Powell, D. A. Autonomicsomatic relationships: Blockade of heart rate and corneoretinal potential responses. Journal of Comparative and Physiological Psychology, 1973, 84, 98-110.

LACEY, B. C., \& LACEY, J. I. Studies of heart rate and other bodily processes in sensorimotor behavior. In P. A. Obrist, A. H. Black, J. Brener, \& L. V. DiCara (Eds.), Cardiovascular psychophysiology: Current issues in response mechanisms, biofeedback, and methodology. Chicago: Aldine, 1974.

Lanier, L. P., \& IsaAcson, R. L. Activity changes related to the location of lesions in the hippocampus. Behavioral Biology, 1975, 13, 59-69.

Овrist, P. A. The cardiovascular-behavioral interaction as it appears today. Psychophysiology, 1976, 13, 95-107.

O'KeEFE, J. O., \& NADEL, L. The hippocampus as a cognitive map. Oxford: Clarendon Press, 1978.

Powell, D. A., \& Jose PH, J. A. Autonomic-somatic interaction and hippocampal theta activity. Journal of Comparative and Physiological Psychology, 1974, 87, 978-986.

Powell, D. A., \& KAZIS, E. Blood pressure and heart rate changes accompanying classical eyeblink conditioning in the rabbit (Oryctolagus cuniculus). Psychophysiology, 1976, 13, 441-447.

Powell, D. A., Lipkin, M., \& Milligan, W. L. Concomitant changes in classically conditioned heart rate and corneoretinal potential discrimination in the rabbit (Oryctolagus cuniculus). Learning and Motivation, 1974, 5, 532-547.

Powell, D. A., Mankowski, D., \& Buchanan, S. Concomitant heart rate and corneoretinal potential conditioning in the rabbit (Oryctolagus cuniculus): Effects of caudate lesions. Physiology \& Behavior, 1978, 20, 143-150.

Powell, D. A., \& Milligan, W. L. Effects of partial and continuous reinforcement on conditioned heart rate and corneoretinal potential responses in the rabbit (Oryctolagus cuniculus). Psychological Record, 1975, 25, 419-426.

Powell, D. A., Milligan, W. L., \& Buchanan, S. L. Orienting and classical conditioning in the rabbit (Oryctolagus cuniculus): Effects of septal area lesions. Physiology \& Behavior, 1976, 17, 955-962.

Powell, E. W., \& Hines, G. Septohippocampal interface. In R. Isaacson \& K. Pribram (Eds.), The hippocampus. New York: Plenum Press, 1975.

Pribram, K. H., \& McGuinness, D. Arousal, activation and effort in the control of attention. Psychological Review, 1975, 82, 116-149.

Sanwald, J. C., Porzio, N. R., Deane, G. E., \& Donovick, P. J. The effects of septal and dorsal hippocampal lesions on the cardiac component of the orienting response. Physiology \& Behavior, 1970, 5, 883-888.

Schmaltz, L. W., \& Theios, J. Acquisition and extinction of a classically conditioned response in hippocampectomized rabbits (Oryctolagus cuniculus). Journal of Comparative and Physiological Psychology, 1972, 79, 328-333.

Schneiderman, N. Response system divergencies in aversive classical conditioning. In A. H. Black \& W. F. Prokasy (Eds.), Classical conditioning II: Current theory and research. New York: Appleton-Century-Crofts, 1972.

Segal, M., \& Olds, J. Activity of units in the hippocampal circuit of the rat during differential classical conditioning. Journal of Comparative and Physiological Psychology, 1973, 82, 195-204.

SoкоLov, E. N. Perception and the conditioned reflex. New York: Macmillan, 1963.

Solomon, P. R. Role of the hippocampus in blocking and conditioned inhibition of the rabbit's nictitating membrane response. Journal of Comparative and Physiological Psychology, 1977, 91, 407-417.

Solomon, P. R., \& Moore, J. W. Latent inhibition and stimulus generalization of the classically conditioned nictitating membrane response in rabbits (Oryctolagus cuniculus) following dorsal hippocampal ablation. Journal of Comparative and Physiological Psychology, 1975, 89, 1192-1203.

Thompson, R. F., Berger, T. W., Cegavske, C. F., Patterson, M. M., Roemer, R. A., Teyler, T. J., \& Young, R. A. The search for the engram. American Psychologist, 1976, 31, 209-227.

VanDercar, D. H., Swadlow, H. A., Elster, A. E., \& Schneiderman, N. Nictitating membrane and corneoretinal transducers for conditioning in rabbits. American Psychologist, $1969,24,262-264$.

Vinogradova, $\mathbf{O}$. S. The hippocampus and the orienting reflex. In E. N. Sokolov \& O. S. Vinogradova (Eds.), Neural mechanisms of the orienting reflex. New York: Wiley, 1975.

(Received for publication April 16, 1980; revision accepted June 8,1980 .) 